\title{
COMMON FIXED POINTS OF SINGLE-VALUED AND MULTIVALUED MAPS
}

\author{
YICHENG LIU, JUN WU, AND ZHIXIANG LI
}

Received 19 June 2005 and in revised form 1 September 2005

We define a new property which contains the property (EA) for a hybrid pair of singleand multivalued maps and give some new common fixed point theorems under hybrid contractive conditions. Our results extend previous ones. As an application, we give a partial answer to the problem raised by Singh and Mishra.

\section{Introduction and preliminaries}

Let $(X, d)$ be a metric space. Then, for $x \in X, A \subset X, d(x, A)=\inf \{d(x, y), y \in A\}$. We denote $C B(X)$ as the class of all nonempty bounded closed subsets of $X$. Let $H$ be the Hausdorff metric with respect to $d$, that is,

$$
H(A, B)=\max \left\{\sup _{x \in A} d(x, B), \sup _{y \in B} d(y, A)\right\},
$$

for every $A, B \in C B(X)$. A self-map $T$ defined on $X$ satisfies Rhoades' contractive definition in following sense: (see [19]) for all $x, y \in X, x \neq y$,

$$
d(T x, T y)<\max \{d(x, y), d(x, T x), d(y, T y), d(x, T y), d(y, T x)\} .
$$

The fixed points theorems for Rhoades-type contraction mapping were investigated by many authors $[1,5,8,10,13,16,22]$ and the more results on this fields can be found in $[2,4,9,11,15,23]$. Hybrid fixed point theory for nonlinear single-valued and multivalued maps is a new development in the domain of contraction-type multivalued theory (see $[3,7,10,12,14,17,18,20]$ and references therein). In 1998, Jungck and Rhoades [12] introduced the notion of weak compatibility to the setting of single-valued and multivalued maps. In [21], Singh and Mishra introduced the notion of (IT)-commutativity for hybrid pair of single-valued and multivalued maps which need not be weakly compatible. Recently, Aamri and El Moutawakil [1] defined a property (EA) for self-maps which contained the class of noncompatible maps. More recently, Kamran [13] extended the property (EA) for a hybrid pair of single- and multivalued maps and generalized the notion of (IT)-commutativity for such pair. 
The aim of this paper is to define a new property which contains the property (EA) for a hybrid pair of single- and multivalued maps and give some new common fixed point theorems under hybrid contractive conditions. As an application, we give an affirmative (half-) answer (Theorem 2.8) to the open problem in [21].

Now we state some known definitions and facts.

Definition 1.1 [12]. Maps $f: X \rightarrow X$ and $T: X \rightarrow C B(X)$ are weakly compatible if they commute at their coincidence points, that is, if $f T x=T f x$ whenever $f x \in T x$.

Definition 1.2 [21]. Maps $f: X \rightarrow X$ and $T: X \rightarrow C B(X)$ are said to be (IT)-commuting at $x \in X$ if $f T x \subset T f x$ whenever $f x \in T x$.

Definition 1.3 [1]. Maps $f, g: X \rightarrow X$ are said to satisfy the property (EA) if there exists a sequence $\left\{x_{n}\right\}$ in $X$ such that $\lim _{n \rightarrow \infty} f x_{n}=\lim _{n \rightarrow \infty} g x_{n}=t \in X$.

Definition 1.4 [13]. Maps $f: X \rightarrow X$ and $T: X \rightarrow C B(X)$ are said to satisfy the property (EA) if there exist a sequence $\left\{x_{n}\right\}$ in $X$, some $t$ in $X$, and $A$ in $C B(X)$ such that

$$
\lim _{n \rightarrow \infty} f x_{n}=t \in A=\lim _{n \rightarrow \infty} T x_{n} .
$$

Definition 1.5 [13]. Let $T: X \rightarrow C B(X)$. The map $f: X \rightarrow X$ is said to be $T$-weakly commuting at $x \in X$ if $f f x \in T f x$.

For the rest of the introduction, we state the following theorem as the prototype in this paper.

TheOREm 1.6 (see [13]). Let $f$ be a self-map of the metric space $(X, d)$ and let $F$ be a map from $X$ into $C B(X)$ such that

(1) $(f, F)$ satisfies the property $(E A)$;

(2) for all $x \neq y$ in $X$,

$$
H(F x, F y)<\max \left\{d(f x, f y), \frac{d(f x, F x)+d(f y, F y)}{2}, \frac{d(f x, F y)+d(f y, F x)}{2}\right\} .
$$

If $f X$ is closed subset of $X$, then

(a) $f$ and $F$ have a coincidence point;

(b) $f$ and $F$ have a common fixed point provided that $f$ is F-weakly commuting at $v$ and $f f v=f v$ for $v \in C(f, F)$, where $C(f, F)=\{x: x$ is a coincidence point of $f$ and $F$ \}.

\section{Main results}

We begin with the following definition.

Definition 2.1. (1) Let $f, g, F, G: X \rightarrow X$. The maps pair $(f, F)$ and $(g, G)$ are said to satisfy the common property (EA) if there exist two sequences $\left\{x_{n}\right\},\left\{y_{n}\right\}$ in $X$ and some $t$ in $X$ such that

$$
\lim _{n \rightarrow \infty} G y_{n}=\lim _{n \rightarrow \infty} F x_{n}=\lim _{n \rightarrow \infty} f x_{n}=\lim _{n \rightarrow \infty} g y_{n}=t \in X .
$$


(2) Let $f, g: X \rightarrow X$ and $F, G: X \rightarrow C B(X)$. The maps pair $(f, F)$ and $(g, G)$ are said to satisfy the common property (EA) if there exist two sequences $\left\{x_{n}\right\},\left\{y_{n}\right\}$ in $X$, some $t$ in $X$, and $A, B$ in $C B(X)$ such that

$$
\lim _{n \rightarrow \infty} F x_{n}=A, \quad \lim _{n \rightarrow \infty} G y_{n}=B, \quad \lim _{n \rightarrow \infty} f x_{n}=\lim _{n \rightarrow \infty} g y_{n}=t \in A \cap B .
$$

Example 2.2. Let $X=[1,+\infty)$ with the usual metric. Define $f, g: X \rightarrow X$ and $F, G: X \rightarrow$ $C B(X)$ by $f(x)=2+x / 3, g(x)=2+x / 2$, and $F(x)=[1,2+x], G(x)=[3,3+x / 2]$ for all $x \in X$. Consider the sequences $\left\{x_{n}\right\}=\{3+1 / n\},\left\{y_{n}\right\}=\{2+1 / n\}$. Clearly, $\lim _{n \rightarrow \infty} F x_{n}=$ $[1,5]=A, \lim _{n \rightarrow \infty} G y_{n}=[3,4]=B, \lim _{n \rightarrow \infty} f x_{n}=\lim _{n \rightarrow \infty} g y_{n}=3 \in A \cap B$. Therefore, $(f, F)$ and $(g, G)$ are said to satisfy the common property (EA).

Theorem 2.3. Let $f, g$ be two self-maps of the metric space $(X, d)$ and let $F$, $G$ be two maps from $X$ into $C B(X)$ such that

(1) $(f, F)$ and $(g, G)$ satisfy the common property $(E A)$;

(2) for all $x \neq y$ in $X$,

$$
H(F x, G y)<\max \left\{d(f x, g y), \frac{d(f x, F x)+d(g y, G y)}{2}, \frac{d(f x, G y)+d(g y, F x)}{2}\right\} .
$$

If $f X$ and $g X$ are closed subsets of $X$, then

(a) $f$ and $F$ have a coincidence point;

(b) $g$ and $G$ have a coincidence point;

(c) $f$ and $F$ have a common fixed point provided that $f$ is F-weakly commuting at $v$ and $f f v=f v$ for $v \in C(f, F)$;

(d) $g$ and $G$ have a common fixed point provided that $g$ is $G$-weakly commuting at $v$ and $g g v=g v$ for $v \in C(g, G)$;

(e) $f, g, F$, and $G$ have a common fixed point provided that both (c) and (d) are true.

Proof. Since $(f, F)$ and $(g, G)$ satisfy the common property (EA), there exist two sequences $\left\{x_{n}\right\},\left\{y_{n}\right\}$ in $X$ and $u \in X, A, B \in C B(X)$ such that

$$
\begin{gathered}
\lim _{n \rightarrow \infty} F x_{n}=A, \quad \lim _{n \rightarrow \infty} G y_{n}=B, \\
\lim _{n \rightarrow \infty} f x_{n}=\lim _{n \rightarrow \infty} g y_{n}=u \in A \cap B .
\end{gathered}
$$

By virtue of $f X$ and $g X$ being closed, we have $u=f v$ and $u=g w$ for some $v, w \in X$. We claim that $f v \in F v$ and $g w \in G w$. Indeed, condition (2) implies that

$$
H\left(F x_{n}, G w\right)<\max \left\{d\left(f x_{n}, g w\right), \frac{d\left(f x_{n}, F x_{n}\right)+d(g w, G w)}{2}, \frac{d\left(f x_{n}, G w\right)+d\left(g w, F x_{n}\right)}{2}\right\}
$$


Taking the limit as $n \rightarrow \infty$, we obtain

$$
\begin{aligned}
H(A, G w) & <\max \left\{d(f v, g w), \frac{d(f v, A)+d(g w, G w)}{2}, \frac{d(f v, G w)+d(g w, A)}{2}\right\} \\
& =\frac{d(g w, G w)}{2} .
\end{aligned}
$$

Since $g w=f v \in A$, it follows from the definition of Hausdorff metric that

$$
d(g w, G w) \leq H(A, G w) \leq \frac{d(g w, G w)}{2}
$$

which implies that $g w \in G w$.

On the other hand, by condition (2) again, we have

$$
H\left(F v, G y_{n}\right)<\max \left\{d\left(f v, g y_{n}\right), \frac{d(f v, F v)+d\left(g y_{n}, G y_{n}\right)}{2}, \frac{d\left(f v, G y_{n}\right)+d\left(g y_{n}, F v\right)}{2}\right\} .
$$

Similarly, we obtain

$$
d(f v, F v) \leq H(F v, B) \leq \frac{d(f v, F v)}{2} .
$$

Hence $f v \in F v$. Thus $f$ and $F$ have a coincidence point $v, g$ and $G$ have a coincidence point $w$. This ends the proofs of part (a) and part (b).

Furthermore, by virtue of condition (c), we obtain $f f v=f v$ and $f f v \in F f v$. Thus $u=f u \in F u$. This proves (c). A similar argument proves (d). Then (e) holds immediately.

Remark 2.4. In Theorem 2.3, if $F, G$ are two maps from $K$ into $C B(X)$, where $K$ is a closed subset of $X$. In this case, it is necessary to assume that $(X, d)$ is a metrically convex metric space. In this direction, many excellent works have appeared (see $[5,21])$.

Corollary 2.5 (see [13, Theorem 3.10]). Let $f$ be a self-map of the metric space $(X, d)$ and let $F$ be a map from $X$ into $C B(X)$ such that

(1) $(f, F)$ satisfies the property $(E A)$;

(2) for all $x \neq y$ in $X$,

$$
H(F x, F y)<\max \left\{d(f x, f y), \frac{d(f x, F x)+d(f y, F y)}{2}, \frac{d(f x, F y)+d(f y, F x)}{2}\right\} .
$$

If $f X$ is closed subset of $X$, then

(a) $f$ and $F$ have a coincidence point;

(b) $f$ and $F$ have a common fixed point provided that $f$ is F-weakly commuting at $v$ and $f f v=f v$ for $v \in C(f, F)$. 
Proof. Let $F=G$ and $f=g$, then the results follow from Theorem 2.3 immediately.

If $f=g$, we can conclude the following corollary.

Corollary 2.6. Let $f$ be a self-map of the metric space $(X, d)$ and let $F, G$ be two maps from $X$ into $C B(X)$ such that

(1) $(f, F)$ and $(f, G)$ satisfy the common property $(E A)$;

(2) for all $x \neq y$ in $X$,

$$
H(F x, G y)<\max \left\{d(f x, f y), \frac{d(f x, F x)+d(f y, G y)}{2}, \frac{d(f x, G y)+d(f y, F x)}{2}\right\} .
$$

If $f X$ is closed subset of $X$, then

(a) $f$, $G$ and $F$ have a coincidence point;

(b) $f, G$ and $F$ have a common fixed point provided that $f$ is both $F$-weakly commuting and $G$-weakly commuting at $v$ and $f f v=f v$ for $v \in C(f, F)$.

If both $F$ and $G$ are single-valued maps in Theorem 2.3, then we have the following corollary.

Corollary 2.7. Let $f, g, F$, and $G$ be four self-maps of the metric space $(X, d)$ such that

(1) $(f, F)$ and $(g, G)$ satisfy the common property $(E A)$;

(2) for all $x \neq y$ in $X$,

$$
d(F x, G y)<\max \left\{d(f x, g y), \frac{d(f x, F x)+d(g y, G y)}{2}, \frac{d(f x, G y)+d(g y, F x)}{2}\right\} .
$$

If $f X$ and $g X$ are closed subsets of $X$, then

(a) $f$ and $F$ have a coincidence point;

(b) $g$ and $G$ have a coincidence point;

(c) $f$ and $F$ have a common fixed point provided that $f$ is F-weakly commuting at $v$ and $f f v=f v$ for $v \in C(f, F)$;

(d) $g$ and $G$ have a common fixed point provided that $g$ is G-weakly commuting at $v$ and $g g v=g v$ for $v \in C(g, G)$;

(e) $f, g, F$, and $G$ have a common fixed point provided that both (c) and (d) are true.

Theorem 2.8. Let $f, g$ be two self-maps of the complete metric space $(X, d)$, let $\lambda \in(0,1)$ be a constant, and let $F, G$ be two maps from $X$ into $C B(X)$ such that for all $x \neq y$ in $X$,

$$
H(F x, G y) \leq \lambda \max \left\{d(f x, g y), d(f x, F x), d(g y, G y), \frac{d(f x, G y)+d(g y, F x)}{2}\right\} .
$$

If $f X$ and $g X$ are closed subsets of $X$ and $F X \subset g X, G X \subset f X$, then

(a) $f$ and $F$ have a coincidence point;

(b) $g$ and $G$ have a coincidence point;

(c) $f$ and $F$ have a common fixed point provided that $f$ is F-weakly commuting at $v$ and $f f v=f v$ for $v \in C(f, F)$; 
(d) $g$ and $G$ have a common fixed point provided that $g$ is G-weakly commuting at $v$ and $g g v=g v$ for $v \in C(g, G)$;

(e) $f, g, F$, and $G$ have a common fixed point provided that both (c) and (d) are true.

Proof. For any given $x_{0} \in X$, by virtue of $F X \subset g X$, there is $x_{1} \in X$ such that $y_{1}=g x_{1} \in$ $F x_{0}$. Now since $F x_{0}$ and $G x_{1}$ are closed sets and $y_{1} \in F x_{0}$, we can find $y_{2} \in G x_{1}$ such that

$$
d\left(y_{1}, y_{2}\right) \leq H\left(F x_{0}, G x_{1}\right)+\lambda .
$$

Since $G X \subset f X$, there exists $x_{2}$ such that $f x_{2}=y_{2} \in G x_{1}$, then we choose $y_{3} \in F x_{2}$ satisfying

$$
d\left(y_{2}, y_{3}\right) \leq H\left(G x_{1}, F x_{2}\right)+\lambda^{2}
$$

and $y_{3}=g x_{3}$ for some $x_{3} \in X$.

We continue this process to obtain a sequence $\left\{y_{n}\right\}$ in $X$ such that

$$
\begin{gathered}
y_{2 n}=f x_{2 n} \in G x_{2 n-1}, \quad y_{2 n+1}=g x_{2 n+1} \in F x_{2 n}, \\
d\left(y_{2 n}, y_{2 n+1}\right) \leq H\left(G x_{2 n-1}, F x_{2 n}\right)+\lambda^{2 n}, \\
d\left(y_{2 n-1}, y_{2 n}\right) \leq H\left(F x_{2 n-2}, G x_{2 n-1}\right)+\lambda^{2 n-1}, \quad n=1,2, \ldots .
\end{gathered}
$$

Let $a_{n}=d\left(y_{n}, y_{n+1}\right)$, then

$$
\begin{aligned}
& a_{2 n}= d\left(y_{2 n}, y_{2 n+1}\right) \leq H\left(G x_{2 n-1}, F x_{2 n}\right)+\lambda^{2 n} \\
& \leq \lambda \max \left\{d\left(f x_{2 n}, g x_{2 n-1}\right), d\left(f x_{2 n}, F x_{2 n}\right), d\left(g x_{2 n-1}, G x_{2 n-1}\right),\right. \\
&\left.\frac{d\left(f x_{2 n}, G x_{2 n-1}\right)+d\left(g x_{2 n-1}, F x_{2 n}\right)}{2}\right\}+\lambda^{2 n} .
\end{aligned}
$$

By $f x_{2 n} \in G x_{2 n-1}$, we have

$$
d\left(g x_{2 n-1}, G x_{2 n-1}\right) \leq d\left(g x_{2 n-1}, f x_{2 n}\right), \quad d\left(f x_{2 n}, F x_{2 n}\right) \leq H\left(G x_{2 n-1}, F x_{2 n}\right) .
$$

Thus, we rewrite (2.17) as

$$
a_{2 n} \leq \lambda \max \left\{d\left(f x_{2 n}, g x_{2 n-1}\right), \frac{d\left(g x_{2 n-1}, F x_{2 n}\right)}{2}\right\}+\lambda^{2 n} .
$$

Hence, we obtain

$$
a_{2 n} \leq \lambda \max \left\{a_{2 n-1}, \frac{a_{2 n-1}+a_{2 n}}{2}\right\}+\lambda^{2 n} .
$$

If $a_{2 n-1} \leq a_{2 n}$ for some $n$, we have $a_{2 n} \leq \lambda^{2 n} /(1-\lambda)$. Otherwise, we get

$$
a_{2 n} \leq \lambda a_{2 n-1}+\lambda^{2 n}
$$


Yicheng Liu et al. 3051

Therefore, by (2.20), we achieve

$$
a_{2 n} \leq \max \left\{\lambda a_{2 n-1}+\lambda^{2 n}, \frac{\lambda^{2 n}}{1-\lambda}\right\} .
$$

On the other hand,

$$
\begin{aligned}
a_{2 n-1} \leq & H\left(G x_{2 n-1}, F x_{2 n-2}\right)+\lambda^{2 n-1} \\
\leq & \lambda \max \left\{d\left(f x_{2 n-2}, g x_{2 n-1}\right), d\left(f x_{2 n-2}, F x_{2 n-2}\right), d\left(g x_{2 n-1}, G x_{2 n-1}\right),\right. \\
& \left.\frac{d\left(f x_{2 n-2}, G x_{2 n-1}\right)+d\left(g x_{2 n-1}, F x_{2 n-2}\right)}{2}\right\}+\lambda^{2 n-1} .
\end{aligned}
$$

Since $g x_{2 n-1} \in F x_{2 n-2}$, we have

$$
\begin{aligned}
& d\left(g x_{2 n-1}, G x_{2 n-1}\right) \leq H\left(G x_{2 n-1}, F x_{2 n-2}\right), \\
& d\left(f x_{2 n-2}, F x_{2 n-2}\right) \leq d\left(g x_{2 n-1}, f x_{2 n-2}\right) .
\end{aligned}
$$

Thus, we obtain

$$
a_{2 n-1} \leq \lambda \max \left\{a_{2 n-2}, \frac{a_{2 n-2}+a_{2 n-1}}{2}\right\}+\lambda^{2 n-1} .
$$

Similarly, we get

$$
a_{2 n-1} \leq \max \left\{\lambda a_{2 n-2}+\lambda^{2 n-1}, \frac{\lambda^{2 n-1}}{1-\lambda}\right\} .
$$

By (2.22) and (2.26), we obtain

$$
a_{n} \leq \max \left\{\lambda a_{n-1}+\lambda^{n}, \frac{\lambda^{n}}{1-\lambda}\right\}, \quad n=1,2, \ldots
$$

It is easy to see that

$$
a_{n} \leq \max \left\{\lambda^{n}\left(a_{0}+n\right), \frac{\lambda^{n}}{1-\lambda}\right\}, \quad n=1,2, \ldots
$$

Thus, there exists $n_{0}>0$ such that for $n \geq n_{0}$,

$$
a_{n} \leq \lambda^{n}\left(a_{0}+n\right) .
$$

Hence $\lim _{n \rightarrow \infty} a_{n}=0$.

In order to prove that $\left\{y_{n}\right\}$ is Cauchy sequence, for any $\varepsilon>0$, we choose a sufficiently large number $N$ such that

$$
\lambda^{N}\left(a_{0}+N\right) \leq \frac{\varepsilon(1-\lambda)}{2}, \quad \lambda^{N} \leq \frac{\varepsilon(1-\lambda)^{2}}{4} .
$$


3052 Common fixed points of hybrid maps

Thus, for any positive integer $k$, we obtain

$$
\begin{aligned}
d\left(y_{N}, y_{N+k}\right) & \leq \sum_{i=0}^{k-1} a_{N+i} \leq \sum_{i=0}^{k-1} \lambda^{N+i}\left(a_{0}+N+i\right) \\
& <\lambda^{N}\left(a_{0}+N\right) \frac{1}{1-\lambda}+\lambda^{N}\left(\sum_{i=0}^{k-1} i \lambda^{i}\right) \\
& <\lambda^{N}\left(a_{0}+N\right) \frac{1}{1-\lambda}+\lambda^{N} \frac{2}{(1-\lambda)^{2}} \leq \varepsilon .
\end{aligned}
$$

This implies that $\left\{y_{n}\right\}$ is a Cauchy sequence. Thus there is $u$ satisfying

$$
\lim _{n \rightarrow \infty} y_{n}=u=\lim _{n \rightarrow \infty} f x_{2 n}=\lim _{n \rightarrow \infty} g x_{2 n+1} .
$$

Since $f X$ and $g X$ are closed, there exist $a, b$ such that $f a=u=g b$. A similar argument proves that

$$
\begin{gathered}
\lim _{n \rightarrow \infty} F x_{2 n}=\lim _{n \rightarrow \infty} G x_{2 n+1}, \\
u \in \lim _{n \rightarrow \infty} F x_{2 n}=\lim _{n \rightarrow \infty} G x_{2 n+1} .
\end{gathered}
$$

Then $(f, F)$ and $(g, G)$ satisfy the common property (EA). The rest of the proof follows Theorem 2.3 immediately, then the proof of Theorem 2.8 is complete.

Corollary 2.9. Let $f, g$ be two self-maps of the complete metric space $(X, d)$, let $\lambda \in(0,1)$ be a constant, and let $F, G$ be two maps from $X$ into $C B(X)$ such that for all $x \neq y$ in $X$,

$$
\begin{aligned}
H(F x, G y) \leq & \alpha d(f x, g y)+\beta \max \{d(f x, F x), d(g y, G y)\} \\
& +\gamma \max \{d(f x, G y)+d(g y, F x), d(f x, F x)+d(g y, G y)\},
\end{aligned}
$$

and $\alpha+\beta+2 \gamma<1$. If $f X$ and $g X$ are closed subsets of $X$ and $F X \subset g X, G X \subset f X$, then

(a) $f$ and $F$ have a coincidence point;

(b) $g$ and $G$ have a coincidence point;

(c) $f$ and $F$ have a common fixed point provided that $f$ is F-weakly commuting at $v$ and $f f v=f v$ for $v \in C(f, F)$;

(d) $g$ and $G$ have a common fixed point provided that $g$ is $G$-weakly commuting at $v$ and $g g v=g v$ for $v \in C(g, G)$;

(e) $f, g, F$, and $G$ have a common fixed point provided that both (c) and (d) are true.

Proof. Let $\lambda=\alpha+\beta+2 \gamma$. Following (2.34) and $\max \{d(f x, F x), d(g y, G y)\} \geq(d(f x, F x)+$ $d(g y, G y)) / 2$, it is easy to see that

$$
H(F x, G y) \leq \lambda \max \left\{d(f x, g y), d(f x, F x), d(g y, G y), \frac{d(f x, G y)+d(g y, F x)}{2}\right\} .
$$

Thus by Theorem 2.8, we arrive to the conclusion in Corollary 2.9. 
The next theorem involves a function $\varphi$. Various conditions on $\varphi$ have been investigated by different authors $[4,6,15,16]$. Let $\varphi: \mathbb{R}^{+} \rightarrow \mathbb{R}^{+}$continue and satisfy the following conditions:

$\left(\mathrm{A}_{1}\right) \varphi$ is nondecreasing on $\mathbb{R}^{+}$,

$\left(\mathrm{A}_{2}\right) 0<\varphi(t)<t$, for each $t \in(0,+\infty)$.

Theorem 2.10. Let $f$, $g$ be two self-maps of the metric space $(X, d)$ and let $F, G: X \rightarrow X$ be two maps from $X$ into $C B(X)$ such that

(1) $(f, F)$ and $(g, G)$ satisfy the common property $(E A)$;

(2) for all $x \neq y$ in $X$,

$$
H(F x, G y) \leq \varphi(\max \{d(f x, g y), d(f x, F x), d(g y, G y), d(f x, G y), d(g y, F x)\}) .
$$

If $f X$ and $g X$ are closed subsets of $X$, then

(a) $f$ and $F$ have a coincidence point;

(b) $g$ and $G$ have a coincidence point;

(c) $f$ and $F$ have a common fixed point provided that $f$ is F-weakly commuting at $v$ and $f f v=f v$ for $v \in C(f, F)$;

(d) $g$ and $G$ have a common fixed point provided that $g$ is G-weakly commuting at $v$ and $g g v=g v$ for $v \in C(g, G)$;

(e) $f, g, F$, and $G$ have a common fixed point provided that both (c) and (d) are true.

Proof. Since $(f, F)$ and $(g, G)$ satisfy the common property (EA), there exist two sequences $\left\{x_{n}\right\},\left\{y_{n}\right\}$ in $X$ and $u \in X, A, B \in C B(X)$ such that

$$
\begin{aligned}
& \lim _{n \rightarrow \infty} F x_{n}=A, \lim _{n \rightarrow \infty} G y_{n}=B, \\
& \lim _{n \rightarrow \infty} f x_{n}=\lim _{n \rightarrow \infty} g y_{n}=u \in A \cap B .
\end{aligned}
$$

By virtue of $f X$ and $g X$ being closed, we have $u=f v$ and $u=g w$ for some $v, w \in X$. We claim that $f v \in F v$ and $g w \in G w$. Indeed, condition (2) implies that

$$
H\left(F x_{n}, G w\right) \leq \varphi\left(\max \left\{d\left(f x_{n}, g w\right), d\left(f x_{n}, F x_{n}\right), d(g w, G w), d\left(f x_{n}, G w\right), d\left(g w, F x_{n}\right)\right\}\right) .
$$

Taking the limit as $n \rightarrow \infty$, we obtain

$$
\begin{aligned}
H(A, G w) & \leq \varphi(\max \{d(f v, g w), d(f v, A), d(g w, G w), d(f v, G w), d(g w, A)\}) \\
& \leq \varphi(d(g w, G w))<d(g w, G w) .
\end{aligned}
$$

Since $g w=f v \in A$, it follows from the definition of Hausdorff metric that

$$
d(g w, G w) \leq H(A, G w)<d(g w, G w),
$$

which implies that $g w \in G w$. 
On the other hand, by condition (2) again, we have

$$
H\left(F v, G y_{n}\right) \leq \varphi\left(\max \left\{d\left(f v, g y_{n}\right), d(f v, F v), d\left(g y_{n}, G y_{n}\right), d\left(f v, G y_{n}\right), d\left(g y_{n}, F v\right)\right\}\right) .
$$

Similarly, we obtain

$$
d(f v, F v) \leq H(F v, B)<d(f v, F v) .
$$

Hence $f v \in F v$. Thus $f$ and $F$ have a coincidence point $v, g$ and $G$ have a coincidence point $w$. This ends the proofs of part (a) and part (b). The rest of proof is similar to the argument of Theorem 2.3.

\section{References}

[1] M. Aamri and D. El Moutawakil, Some new common fixed point theorems under strict contractive conditions, J. Math. Anal. Appl. 270 (2002), no. 1, 181-188.

[2] A. Ahmad and M. Imdad, Some common fixed point theorems for mappings and multi-valued mappings, J. Math. Anal. Appl. 218 (1998), no. 2, 546-560.

[3] J. S. Bae, Fixed point theorems for weakly contractive multivalued maps, J. Math. Anal. Appl. 284 (2003), no. 2, 690-697.

[4] D. W. Boyd and J. S. W. Wong, On nonlinear contractions, Proc. Amer. Math. Soc. 20 (1969), no. $2,458-464$.

[5] Lj. B. Ćirić and J. S. Ume, Multi-valued non-self-mappings on convex metric spaces, Nonlinear Anal. 60 (2005), no. 6, 1053-1063.

[6] D. Downing and W. A. Kirk, A generalization of Caristi's theorem with applications to nonlinear mapping theory, Pacific J. Math. 69 (1977), no. 2, 339-346.

[7] R. Espínola and W. A. Kirk, Set-valued contractions and fixed points, Nonlinear Anal. 54 (2003), no. 3, 485-494.

[8] M. Frigon, Fixed point results for generalized contractions in gauge spaces and applications, Proc. Amer. Math. Soc. 128 (2000), no. 10, 2957-2965.

[9] A. Granas and J. Dugundji, Fixed Point Theory, Springer Monographs in Mathematics, Springer, New York, 2003.

[10] T. Hicks and B. E. Rhoades, Fixed points and continuity for multivalued mappings, Int. J. Math. Math. Sci. 15 (1992), no. 1, 15-30.

[11] V. I. Istrăţescu, Fixed Point Theory: An Introduction, Mathematics and Its Applications, vol. 7, D.Reidel, Dordrecht, 1981.

[12] G. Jungck and B. E. Rhoades, Fixed points for set valued functions without continuity, Indian J. Pure Appl. Math. 29 (1998), no. 3, 227-238.

[13] T. Kamran, Coincidence and fixed points for hybrid strict contractions, J. Math. Anal. Appl. 299 (2004), no. 1, 235-241.

[14] T.-C. Lim, A fixed point theorem for weakly inward multivalued contractions, J. Math. Anal. Appl. 247 (2000), no. 1, 323-327.

[15] _ On characterizations of Meir-Keeler contractive maps, Nonlinear Anal. Ser. A: Theory Methods 46 (2001), no. 1, 113-120.

[16] Y. C. Liu and Zh. X. Li, Schaefer Type Theorem and Periodic Solutions of Evolution Equations, to appear in J. Math. Anal. Appl.

[17] S. V. R. Naidu, Fixed point theorems for a broad class of multimaps, Nonlinear Anal. 52 (2003), no. 3, 961-969.

[18] P. Oliveira, Two results on fixed points, Nonlinear Anal. 47 (2001), no. 4, 2703-2717. 
[19] B. E. Rhoades, A comparison of various definitions of contractive mappings, Trans. Amer. Math. Soc. 226 (1977), 257-290.

[20] N. Shahzad, Coincidence points and R-subweakly commuting multivalued maps, Demonstratio Math. 36 (2003), no. 2, 427-431.

[21] S. L. Singh and S. N. Mishra, Coincidences and fixed points of nonself hybrid contractions, J. Math. Anal. Appl. 256 (2001), no. 2, 486-497.

[22] T. Suzuki, Generalized Caristi's fixed point theorems by Bae and others, J. Math. Anal. Appl. 302 (2005), no. 2, 502-508.

[23] T. Suzuki and W. Takahashi, Fixed point theorems and characterizations of metric completeness, Topol. Methods Nonlinear Anal. 8 (1996), no. 2, 371-382 (1997).

Yicheng Liu: Department of Mathematics and System Sciences, College of Science, National University of Defense Technology, Changsha 410073, China

E-mail address: liuyc2001@hotmail.com

Jun Wu: College of Mathematics and Econometrics, Hunan University, Changsha 410082, China E-mail address: junwmath@hotmail.com

Zhixiang Li: Department of Mathematics and System Sciences, College of Science, National University of Defense Technology, Changsha 410073, China

E-mail address: zhxli02@yahoo.com.cn 


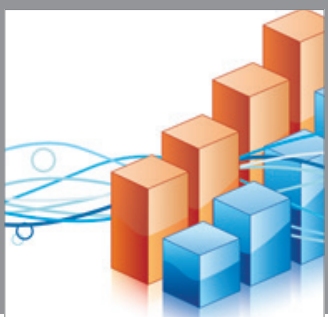

Advances in

Operations Research

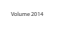

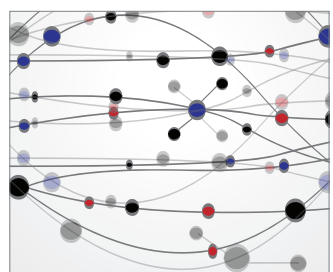

\section{The Scientific} World Journal
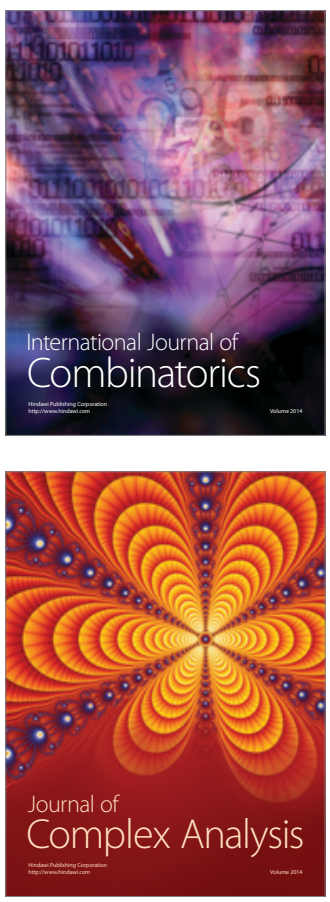

International Journal of

Mathematics and

Mathematical

Sciences
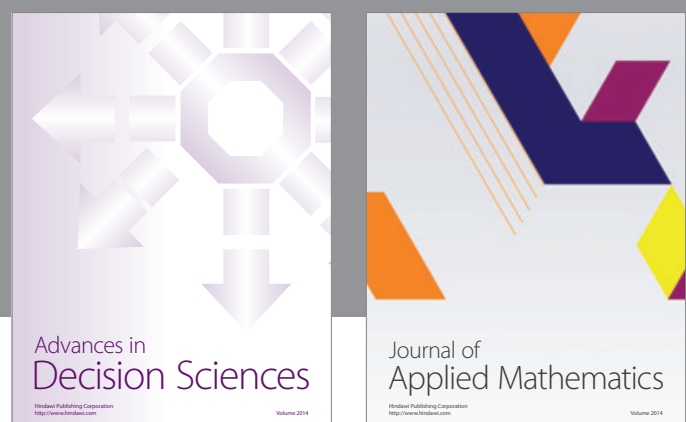

Journal of

Applied Mathematics
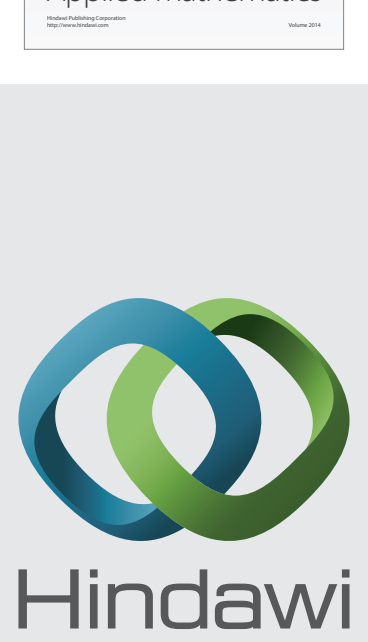

Submit your manuscripts at http://www.hindawi.com
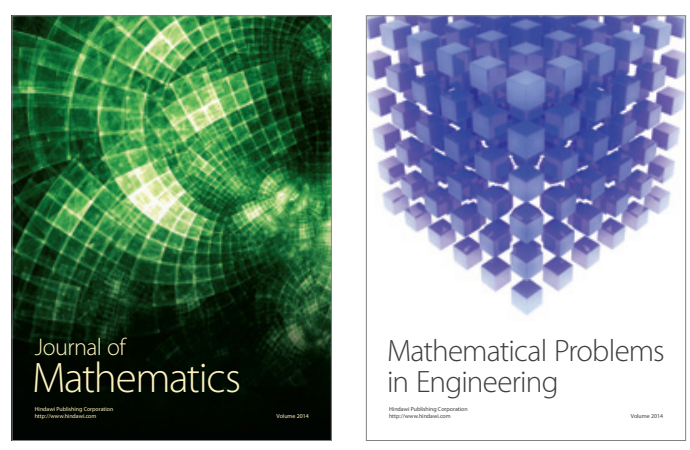

Mathematical Problems in Engineering
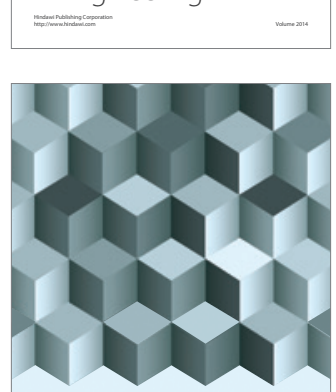

Journal of

Function Spaces
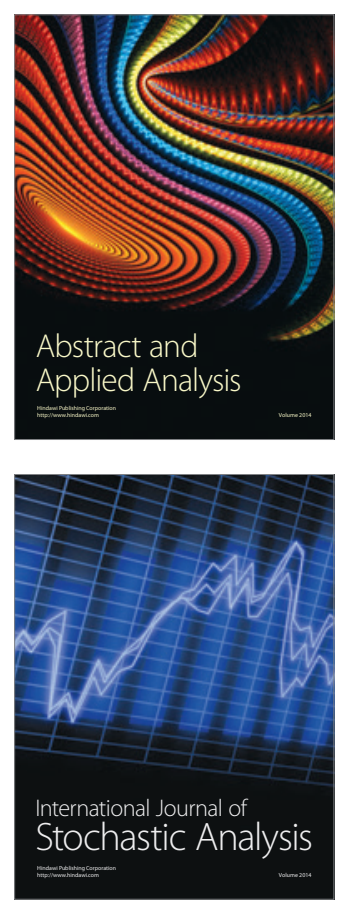

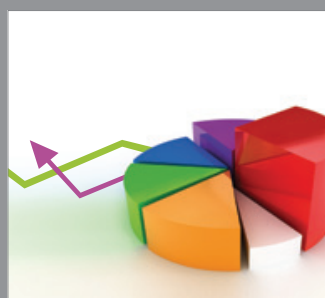

ournal of

Probability and Statistics

Promensencen
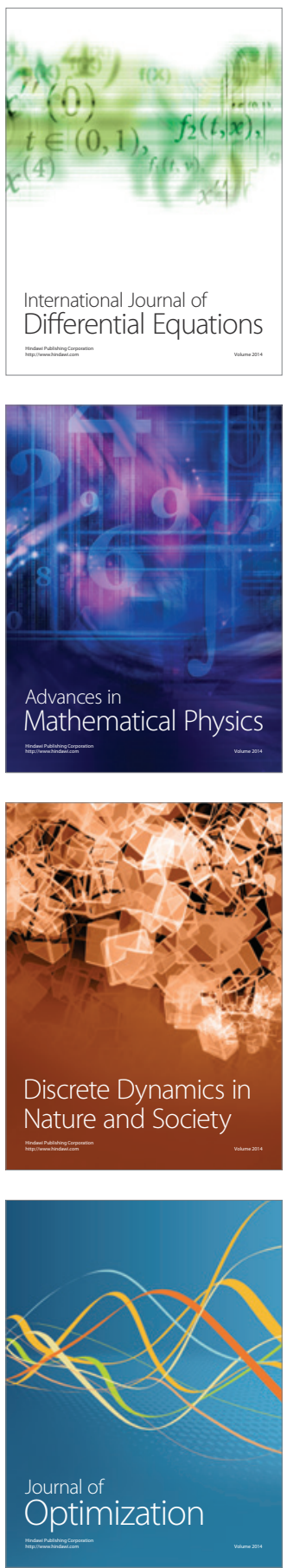